ZMP-HH/16-21

Hamburger Beiträge zur Mathematik Nr. 618

\title{
AN EQUIVALENCE BETWEEN SEMISIMPLE SYMMETRIC FROBENIUS ALGEBRAS AND CALABI-YAU CATEGORIES
}

\author{
JAN HESSE
}

\begin{abstract}
We show that the bigroupoid of semisimple symmetric Frobenius algebras over an algebraically closed field and the bigroupoid of Calabi-Yau categories are equivalent. To this end, we construct a trace on the category of finitely-generated representations of a symmetric, semisimple Frobenius algebra, given by the composite of the Frobenius form with the Hattori-Stallings trace.
\end{abstract}

\section{INTRODUCTION}

The starting point for this paper is the weak 2-functor sending an algebra to its category of representations. If $\mathrm{Alg}_{2}$ denotes the Morita bicategory and $\mathrm{Vect}_{2}$ is the bicategory of linear categories, the weak 2-functor Rep : $\mathrm{Alg}_{2}^{\mathrm{fd}} \rightarrow \mathrm{Vect}_{2}^{\mathrm{fd}}$ sending a semisimple algebra to its category of finitely-generated modules is an equivalence between the fully-dualizable objects of the bicategories $\mathrm{Alg}_{2}$ and $\mathrm{Vect}_{2}$, cf. BDSV15, Appendix A].

In this paper, we endow the objects of $\mathrm{Alg}_{2}^{\mathrm{fd}}$ with the additional structure of a symmetric Frobenius algebra. We show that the category of finitely-generated representations of a semisimple symmetric Frobenius algebra carries a canonical structure of a Calabi-Yau category as considered in [MS06]. The Calabi-Yau structure on the representation category is given by the composite of the Hattori-Stallings trace with the Frobenius form. This allows us to construct a 2 -functor

$$
\operatorname{Rep}^{\mathrm{fg}}: \text { Frob } \rightarrow \mathrm{CY}
$$

between the bigroupoid of semisimple symmetric Frobenius algebras Frob, and the bigroupoid of CalabiYau categories CY. Our main result in theorem 4.3 shows that this 2 -functor is an equivalence of bigroupoids.

This result is related to topological quantum field theories as follows: the Baez-Dolan cobordism hypothesis, proved by Lurie [Lur09] in an $(\infty, n)$-categorical setting, asserts that a framed, fully-extended, topological quantum field theory is classified by its value on the positively framed point. However, one needs more data to classify oriented theories, which is given by the datum of an homotopy fixed point of a certain $S O(n)$-action on the target category.

In 2-dimensions, it is claimed in [FHLT10] that the structure of an $S O(2)$ fixed point on the fullydualizable objects of $\mathrm{Alg}_{2}$ is given by a semisimple symmetric Frobenius algebra. Furthermore, SchommerPries showed in SP09 that the bigroupoid Frob classifies fully extended oriented field theories with target $\mathrm{Alg}_{2}$. Thus, semisimple symmetric Frobenius algebras should correspond to homotopy fixed points of an $S O(2)$-action on fully-dualizable objects of $\mathrm{Alg}_{2}$.

In her thesis, Davidovich Dav11 observed that the $S O(2)$-action on $\mathrm{Alg}_{2}^{\mathrm{fd}}$ is trivializable. Thus, it suffices to consider the trivial $S O(2)$-action. This approach is taken in [HSV16, where the bigroupoids of homotopy fixed points of the trivial $S O(2)$-action on $\mathrm{Alg}_{2}^{\mathrm{fd}}$ and $\mathrm{Vect}_{2}^{\mathrm{fd}}$ are computed in a purely bicategorical setting, and respectively found to be equivalent to Frob and CY. Thus, combining the results above, one should expect that the two bigroupoids Frob and CY are equivalent. In this paper, we establish the equivalence directly, without referring to the cobordism hypothesis and homotopy fixed points.

The paper is organized as follows: in section 2 we recall the definition of the bicategory Frob of semisimple, symmetric Frobenius algebras, compatible Morita contexts and intertwiners, and of the bicategory CY of Calabi-Yau categories.

In section 3 , we construct a weak 2 -functor Rep ${ }^{\mathrm{fg}}$ which sends a semisimple symmetric Frobenius algebra to its category of finitely-generated modules. We endow this category of representations with the Calabi-Yau structure given by the composite of the Frobenius form with the Hattori-Stallings trace in 
definition 3.3 , and show in theorem 4.3 that this functor is an equivalence of bicategories. Section 4 is devoted to the proof of theorem 4.3 .

Throughout the paper we use the following conventions: we will work over an algebraically closed field $\mathbb{K}$. All Frobenius algebras appearing will be symmetric.

\section{ACKNOWLEDGMENTS}

I would like to thank Christoph Schweigert and Alessandro Valentino for inspiring discussions, and Ingo Runkel for pointing out lemma 2.11. I would also like to thank the referee for her/his very thorough report, which helped to sharpen the focus of this paper. The author is supported by the RTG 1670 "Mathematics inspired by String Theory and Quantum Field Theory".

\section{Frobenius algebras and Calabi-Yau categories}

For the convenience of the reader, we recall the definitions of compatible Morita contexts between symmetric Frobenius algebras. This material has already appeared in [SP09] and [HSV16].

\subsection{The bicategory of symmetric Frobenius algebras.}

Definition 2.1. A Frobenius algebra $(A, \lambda)$ over a field $\mathbb{K}$ consists of an associative, unital $\mathbb{K}$-algebra $A$, together with a linear map $\lambda: A \rightarrow \mathbb{K}$, so that the pairing

$$
\begin{aligned}
A \otimes_{\mathbb{K}} A & \rightarrow \mathbb{K} \\
a \otimes b & \mapsto \lambda(a b)
\end{aligned}
$$

is non-degenerate. A Frobenius algebra is called symmetric if $\lambda(a b)=\lambda(b a)$ for all $a$ and $b$ in $A$.

Definition 2.2. Let $A$ and $B$ be two algebras. A Morita context $\mathcal{M}$ consists of a quadruple $\mathcal{M}:=$ $\left({ }_{B} M_{A},{ }_{A} N_{B}, \varepsilon, \eta\right)$, where ${ }_{B} M_{A}$ is a $(B, A)$-bimodule, ${ }_{A} N_{B}$ is an $(A, B)$-bimodule, and

$$
\begin{gathered}
\varepsilon:{ }_{A} N \otimes{ }_{B} M_{A} \rightarrow{ }_{A} A_{A} \\
\eta:{ }_{B} B_{B} \rightarrow{ }_{B} M \otimes{ }_{A} N_{B}
\end{gathered}
$$

are isomorphisms of bimodules, so that the two diagrams

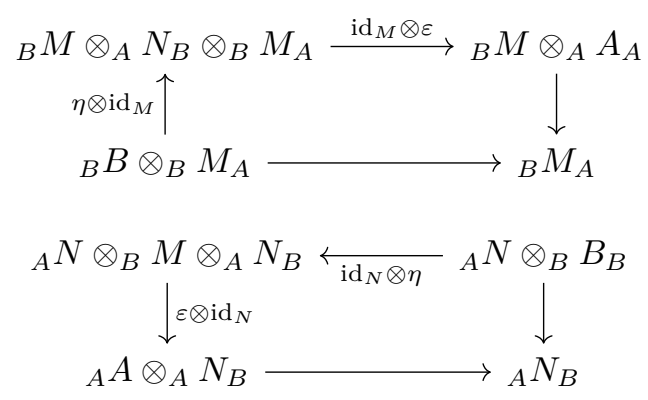

commute.

These two conditions are not independent from each other, as the next lemma proves.

Lemma 2.3 ([Bas68, Lemma 3.3]). In the situation of definition 2.2, diagram (2.3) commutes if and only if diagram (2.4) commutes.

Note that Morita contexts are the adjoint 1-equivalences in the bicategory $\mathrm{Alg}_{2}$ of algebras, bimodules and intertwiners. These form a category, where the morphisms are given by the following:

Definition 2.4. Let $\mathcal{M}:=\left({ }_{B} M_{A},{ }_{A} N_{B}, \varepsilon, \eta\right)$ and $\mathcal{M}^{\prime}:=\left({ }_{B} M^{\prime}{ }_{A},{ }_{A} N^{\prime}{ }_{B}, \varepsilon^{\prime}, \eta^{\prime}\right)$ be two Morita contexts between two algebras $A$ and $B$. A morphism of Morita contexts consists of a morphism of $(B, A)$ bimodules $f: M \rightarrow M^{\prime}$ and a morphism of $(A, B)$-bimodules $g: N \rightarrow N^{\prime}$, so that the two diagrams
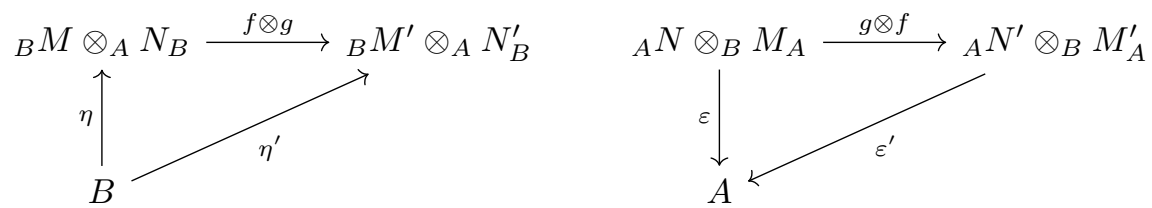

commute. 
If the algebras in question have the additional structure of a symmetric Frobenius form $\lambda: A \rightarrow \mathbb{K}$, we would like to formulate a compatibility condition between the Morita context and the Frobenius forms. The next lemma helps us to do that:

Lemma 2.5 ([SP09, Lemma 3.71] or [HSV16, Lemma 2.3]). Let $A$ and $B$ be two algebras, and let $\left({ }_{B} M_{A},{ }_{A} N_{B}, \varepsilon, \eta\right)$ be a Morita context between $A$ and $B$. Then, there is a canonical isomorphism of vector spaces

$$
\begin{aligned}
f: A /[A, A] & \rightarrow B /[B, B] \\
{[a] } & \mapsto \sum_{i, j}\left[\eta^{-1}\left(m_{j} . a \otimes n_{i}\right)\right]
\end{aligned}
$$

where $n_{i}$ and $m_{j}$ are defined by

$$
\varepsilon^{-1}\left(1_{A}\right)=\sum_{i, j} n_{i} \otimes m_{j} \in N \otimes_{B} M
$$

The isomorphism $f$ described in Lemma 2.5 allows to introduce the following relevant definition.

Definition 2.6. Let $\left(A, \lambda^{A}\right)$ and $\left(B, \lambda^{B}\right)$ be two symmetric Frobenius algebras, and let $\left({ }_{B} M_{A},{ }_{A} N_{B}, \varepsilon, \eta\right)$ be a Morita context between $A$ and $B$. Since the Frobenius algebras are symmetric, the Frobenius forms necessarily factor through $A /[A, A]$ and $B /[B, B]$. We call the Morita context compatible with the Frobenius forms, if the diagram

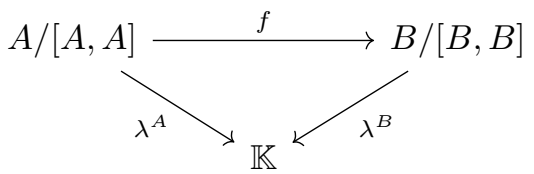

commutes. Using the notation from lemma 2.5 this means that

$$
\lambda^{A}([a])=\sum_{i} \lambda^{B}\left(\left[\eta^{-1}\left(m_{i} \cdot a \otimes n_{i}\right)\right]\right)
$$

for all $a \in A$.

Definition 2.7. Let Frob be the bicategory where

- objects are given by semisimple, symmetric Frobenius algebras,

- 1-morphisms are given by compatible Morita contexts, as in definition 2.6.

- 2-morphisms are given by isomorphisms of Morita contexts.

Note that Frob has got the structure of a symmetric monoidal bigroupoid, where the monoidal product is given by the tensor product over the ground field, which is the monoidal unit.

2.2. The bicategory of Calabi-Yau categories. Another main player of this paper are Calabi-Yau categories, which we define next.

Let $\mathbb{K}$ be a field, and let Vect be the category of $\mathbb{K}$-vector spaces. Recall the following terminology: a linear category is an abelian category with a compatible enrichment over Vect. A linear functor is a right-exact additive functor which is also a functor of Vect-enriched categories.

Definition 2.8. Following [BDSV15, Appendix A], we call a linear category $\mathcal{C}$ finite, if

(1) there are only finitely many isomorphism classes of simple objects of $\mathcal{C}$,

(2) the category $\mathcal{C}$ has enough projectives,

(3) every object of $\mathcal{C}$ has finite length, and

(4) the Hom-spaces of $\mathcal{C}$ are finite-dimensional.

Definition 2.9. A Calabi-Yau category $\left(\mathcal{C}, \operatorname{tr}^{\mathcal{C}}\right)$ is a linear, finite, semisimple category $\mathcal{C}$, together with a family of linear maps

$$
\operatorname{tr}_{c}^{\mathcal{C}}: \operatorname{End}_{\mathcal{C}}(c) \rightarrow \mathbb{K}
$$

for each object $c$ of $\mathcal{C}$, so that:

(1) for each $f \in \operatorname{Hom}_{\mathcal{C}}(c, d)$ and for each $g \in \operatorname{Hom}_{\mathcal{C}}(d, c)$, we have that

$$
\operatorname{tr}_{c}^{\mathcal{C}}(g \circ f)=\operatorname{tr}_{d}^{\mathcal{C}}(f \circ g),
$$


(2) for all objects $c$ and $d$ of $\mathcal{C}$, the induced pairing

$$
\begin{aligned}
\langle-,-\rangle_{\mathcal{C}}: \operatorname{Hom}_{\mathcal{C}}(c, d) \otimes_{\mathbb{K}} \operatorname{Hom}_{\mathcal{C}}(d, c) & \rightarrow \mathbb{K} \\
f \otimes g & \mapsto \operatorname{tr}_{c}^{\mathcal{C}}(g \circ f)
\end{aligned}
$$

is a non-degenerate pairing of $\mathbb{K}$-vector spaces.

We will call the collection of morphisms $\operatorname{tr}_{c}^{\mathcal{C}}$ a trace on $\mathcal{C}$. Note that an equivalent way of defining a Calabi-Yau structure on a linear category $\mathcal{C}$ is by specifying a natural isomorphism

$$
\operatorname{Hom}_{\mathcal{C}}(c, d) \rightarrow \operatorname{Hom}_{\mathcal{C}}(d, c)^{*},
$$

cf. [Sch13, Proposition 4.1].

Remark 2.10. A Calabi Yau category with one object is a semisimple symmetric Frobenius algebra. More generally, the space of all endomorphisms of an object of a Calabi-Yau category has got the structure of a semisimple symmetric Frobenius algebra.

We begin with two preparatory lemmas:

Lemma 2.11. Let $\left(\mathcal{C}, \operatorname{tr}^{\mathcal{C}}\right)$ be a Calabi-Yau category. Then, the trace is automatically additive: for each $f \in \operatorname{End}_{\mathcal{C}}(x)$ and each $g \in \operatorname{End}_{\mathcal{C}}(y)$, we have that

$$
\operatorname{tr}_{x \oplus y}^{\mathcal{C}}(f \oplus g)=\operatorname{tr}_{x}^{\mathcal{C}}(f)+\operatorname{tr}_{y}^{\mathcal{C}}(g) .
$$

Proof. Denote by $p_{x}: x \oplus y \leftrightarrow x: \iota_{x}$ and by $p_{y}: x \oplus y \leftrightarrow y: \iota_{y}$ the canonical projections and inclusions. Then,

$$
\operatorname{id}_{x \oplus y}=\iota_{x} \circ p_{x}+\iota_{y} \circ p_{y} .
$$

Then, by the linearity and cyclicity of the trace,

$$
\begin{aligned}
\operatorname{tr}_{x \oplus y}^{\mathcal{C}}(f+g) & =\operatorname{tr}_{x \oplus y}^{\mathcal{C}}\left((f+g) \circ\left(\iota_{x} \circ p_{x}+\iota_{y} \circ p_{y}\right)\right) \\
& =\operatorname{tr}_{x \oplus y}^{\mathcal{C}}\left((f+g) \circ \iota_{x} \circ p_{x}\right)+\operatorname{tr}_{x \oplus y}^{\mathcal{C}}\left((f+g) \circ \iota_{y} \circ p_{y}\right) \\
& =\operatorname{tr}_{x}^{\mathcal{C}}\left(p_{x} \circ(f+g) \circ \iota_{x}\right)+\operatorname{tr}_{y}^{\mathcal{C}}\left(p_{y} \circ(f+g) \circ \iota_{y}\right) \\
& =\operatorname{tr}_{x}^{\mathcal{C}}(f)+\operatorname{tr}_{y}^{\mathcal{C}}(g) .
\end{aligned}
$$

Adapting the proof of [Sta65, Section 1] to the setting of linear categories establishes the following lemma:

Lemma 2.12. Let $\left(\mathcal{C}, \operatorname{tr}^{\mathcal{C}}\right)$ be a Calabi-Yau category, and let $x_{1}, \ldots, x_{n}$ be objects of $\mathcal{C}$. Let $x:=\oplus_{i=1}^{n} x_{i}$, and let $f \in \operatorname{End}_{\mathcal{C}}(x)$. Since $\mathcal{C}$ is an additive category, we may write the morphism $f$ in matrix form as

$$
f=\left(\begin{array}{ccc}
f_{11} & \ldots & f_{1 n} \\
\vdots & & \vdots \\
f_{n 1} & \ldots & f_{n n}
\end{array}\right)
$$

where the entries $f_{i j}$ are morphisms $f_{i j} \in \operatorname{Hom}_{\mathcal{C}}\left(x_{j}, x_{i}\right)$. Then,

$$
\operatorname{tr}_{x}^{\mathcal{C}}(f)=\sum_{i=1}^{n} \operatorname{tr}_{x_{i}}^{\mathcal{C}}\left(f_{i i}\right) .
$$

Definition 2.13. Let $\left(\mathcal{C}, \operatorname{tr}^{\mathcal{C}}\right)$ and $\left(\mathcal{D}, \operatorname{tr}^{\mathcal{D}}\right)$ be two Calabi-Yau categories. A linear functor $F: \mathcal{C} \rightarrow \mathcal{D}$ is called a Calabi-Yau functor, if

$$
\operatorname{tr}_{c}^{\mathcal{C}}(f)=\operatorname{tr}_{F(c)}^{\mathcal{D}}(F(f))
$$

for each $c \in \mathrm{Ob}(\mathcal{C})$ and each $f \in \operatorname{End}_{\mathcal{C}}(c)$. Equivalently, one may require that

$$
\langle F f, F g\rangle_{\mathcal{D}}=\langle f, g\rangle_{\mathcal{C}}
$$

for every pair of morphisms $f: c \rightarrow c^{\prime}$ and $g: c^{\prime} \rightarrow c$ in $\mathcal{C}$.

If $F, G: \mathcal{C} \rightarrow \mathcal{D}$ are two Calabi-Yau functors between Calabi-Yau categories, a natural transformation of Calabi-Yau functors is just an ordinary natural transformation.

Definition 2.14. Let CY be the symmetric monoidal bigroupoid of Calabi-Yau categories, consisting of objects: Calabi-Yau categories,

1-morphisms: Equivalences of Calabi-Yau categories as in definition 2.13 
2-morphisms: Natural isomorphisms.

The monoidal structure of the bigroupoid CY is given by the Deligne tensor product of finite, abelian categories.

Lemma 2.15. Let $\mathcal{C}$ be a finite semisimple linear category with $n$ simple objects over a field $\mathbb{K}$. Then, $\mathcal{C}$ has got a structure of a Calabi-Yau category. Furthermore, the set of Calabi-Yau structures on $\mathcal{C}$ stands in bijection to $\left(\mathbb{K}^{*}\right)^{n}$.

Proof. If $\mathcal{C}$ has got the structure of a Calabi-Yau category, the trace $\operatorname{tr}^{\mathcal{C}}$ will be additive by lemma 2.12 . Hence, the trace $\operatorname{tr}^{\mathcal{C}}$ is uniquely determined by the endomorphism algebras of the simple objects. If $X$ is a simple object of $\mathcal{C}$, Schur's lemma shows that $\operatorname{End}_{\mathcal{C}}(X) \cong \mathbb{K}$ as vector spaces, since the ground field $\mathbb{K}$ was assumed to be algebraically closed and $\mathcal{C}$ is finite. One now checks that choosing

$$
\operatorname{tr}_{X}^{\mathcal{C}}: \operatorname{End}_{\mathcal{C}}(X) \cong \mathbb{K} \rightarrow \mathbb{K}
$$

to be the identity for every simple object $X$ indeed defines the structure of a Calabi-Yau category on $\mathcal{C}$. This shows the first claim.

Now note that for a simple object $X$, due to its symmetry the $\operatorname{trace} \operatorname{tr}_{X}^{\mathcal{C}}$ is unique up to multiplication with an invertible central element in $Z\left(\operatorname{End}_{\mathcal{C}}(X)\right) \cong \mathbb{K}$. Thus, the $\operatorname{trace} \operatorname{tr}_{X}^{\mathcal{C}}$ on $\operatorname{End}_{\mathcal{C}}(X)$ is unique up to a non-zero element in $\mathbb{K}$. Taking direct sums now shows the second claim.

\section{Constructing the equivalence}

The purpose of this section is to construct a weak 2 -functor Rep ${ }^{\mathrm{fg}}$ : Frob $\rightarrow$ CY, which sends a semisimple, symmetric Frobenius algebra to its category of finitely generated modules.

3.1. A Calabi-Yau structure on the representation category of a Frobenius algebra. First, we will show that the category of finitely generated modules over a semisimple symmetric Frobenius algebra has the structure of a Calabi-Yau category in the sense of definition 2.9 and thus construct Rep ${ }^{\mathrm{fg}}$ on objects.

Let us first recall some standard material about finitely generated and projective $R$-modules. If $M$ is a left $A$-module, the dual module $M^{*}:=\operatorname{Hom}_{A}(M, A)$ is a right $A$-module with right action given by $(f . a)(m):=f(m) . a$.

Lemma 3.1 (Dual basis lemma). Let $R$ be a commutative ring, let $A$ be a $R$-algebra, and let $P$ be a left A-module. The following are equivalent:

(1) The module $P$ is finitely generated and projective.

(2) There are $f_{1}, \ldots, f_{n} \in P^{*}$ and $p_{1}, \ldots, p_{n} \in P$ (sometimes called dual- or projective basis of $P$ ) so that

$$
x=\sum_{i=1}^{n} f_{i}(x) \cdot p_{i}
$$

for all $x \in P$.

(3) The map

$$
\begin{aligned}
\Psi_{P, P}: P^{*} \otimes_{A} P & \rightarrow \operatorname{End}_{A}(P) \\
f \otimes p & \mapsto(x \mapsto f(x) \cdot p)
\end{aligned}
$$

is an isomorphism of R-modules.

(4) For any other left $A$-module $M$, the map

$$
\begin{aligned}
\Psi_{P, M}: P^{*} \otimes_{A} M & \rightarrow \operatorname{Hom}_{A}(P, M) \\
f \otimes m & \mapsto(x \mapsto f(x) . m)
\end{aligned}
$$

is an isomorphism of R-modules.

Proof. The equivalence of (1) and (2) is proven in [Lam12, Lemma 2.9]. The implication (1) $\Rightarrow(4)$ is AW92, Proposition 2.32].

$(4) \Rightarrow(3)$ is trivial, since we may choose $M:=P$.

$(3) \Rightarrow(2)$ : Suppose that $\Psi_{P, P}: P^{*} \otimes_{A} P \rightarrow \operatorname{End}_{A}(P)$ is an isomorphism. Then, a quick calculation confirms that $\Psi_{P, P}^{-1}\left(\operatorname{id}_{P}\right)$ is a dual basis.

Corollary 3.2. Let $A$ be a semisimple algebra, and let $M$ be a finitely generated A-module. Then, $\Psi_{M, M}: M^{*} \otimes_{A} M \rightarrow \operatorname{End}_{A}(M)$ is an isomorphism. 
Proof. As every $A$-module is projective by assumption, the corollary follows directly from lemma 3.1 .

This corollary enables us to define a trace for finitely-generated modules over a semisimple symmetric Frobenius algebra.

Definition 3.3. Let $(A, \lambda)$ be a semisimple symmetric Frobenius algebra with Frobenius form $\lambda: A \rightarrow \mathbb{K}$. Let $M$ be a finitely-generated left $A$-module. Denote by

$$
\text { ev : } \begin{aligned}
M^{*} \otimes_{A} M & \rightarrow A \\
f \otimes m & \mapsto f(m)
\end{aligned}
$$

the evaluation.

Since $M$ is finitely generated, the map $\Psi_{M, M}: \operatorname{End}_{A}(M) \rightarrow M^{*} \otimes_{A} M$ is an isomorphism by corollary 3.2 We define a trace $\operatorname{tr}_{M}^{\lambda}: \operatorname{End}_{A}(M) \rightarrow \mathbb{K}$ by the composition

$$
\operatorname{tr}_{M}^{\lambda}: \operatorname{End}_{A}(M) \stackrel{\Psi_{M, M}^{-1}}{\longrightarrow} M^{*} \otimes_{A} M \stackrel{\mathrm{ev}}{\longrightarrow} A \stackrel{\lambda}{\rightarrow} \mathbb{K}
$$

Remark 3.4. As defined here, the $\operatorname{trace}_{\operatorname{tr}_{M}^{\lambda}}$ is the composition of the Hattori-Stallings trace with the Frobenius form $\lambda$. For more on the Hattori-Stallings trace, see [Hat65], [Sta65] and [Bas76].

Example 3.5. Let $(A, \lambda)$ be a semisimple symmetric Frobenius algebra. Suppose that $F$ is a free $A$-module with basis $e_{1}, \ldots, e_{n}$. Then,

$$
\operatorname{tr}_{F}^{\lambda}\left(\operatorname{id}_{F}\right)=n \lambda\left(1_{A}\right)
$$

Example 3.6. As a second example, and let $A:=M_{n}(\mathbb{K})$ be the algebra of $n \times n$-matrices over $\mathbb{K}$ with Frobenius form $\lambda$ given by the usual trace of matrices. Then, $M:=\mathbb{K}^{n}$ is a projective (but not free), simple $A$-module. We claim:

$$
\operatorname{tr}_{M}^{\lambda}\left(\mathrm{id}_{M}\right)=1
$$

Indeed, let $e_{1}, \ldots, e_{n}$ be a vector space basis of $\mathbb{K}^{n}$. This basis also generates $\mathbb{K}^{n}$ as an $A$-module. Define for each $1 \leq i \leq n$ a $\mathbb{K}$-linear map $f_{i}^{*}: \mathbb{K}^{n} \rightarrow M_{n}(\mathbb{K})=A$ by setting

$$
f_{i}^{*}\left(e_{k}\right):=\delta_{i, 1} E_{k, 1},
$$

where $E_{k, 1}$ is the square matrix with $(k, 1)$-entry given by one and zero otherwise. A short calculation confirms that the $f_{i}^{*}$ are even morphisms of $A$-modules. Next, we claim that

$$
\Psi_{M, M}^{-1}=\sum_{i=1}^{n} f_{i}^{*} \otimes e_{i} \in M^{*} \otimes_{A} M .
$$

Indeed,

$$
\begin{aligned}
\Psi_{M, M}\left(\sum_{i=1}^{n} f_{i}^{*} \otimes e_{i}\right)\left(e_{k}\right) & =\sum_{i=1}^{n} f_{i}^{*}\left(e_{k}\right) \cdot e_{i} \\
& =\sum_{i=1}^{n} \delta_{i, 1} E_{k, 1} e_{i} \\
& =E_{k, 1} e_{1}=e_{k}
\end{aligned}
$$

Thus,

$$
\operatorname{tr}_{M}^{\lambda}\left(\operatorname{id}_{M}\right)=\lambda\left(\sum_{i=1}^{n} f_{i}^{*}\left(e_{i}\right)\right)=\lambda\left(\sum_{i=1}^{n} E_{i, 1} \delta_{1, i}\right)=\lambda\left(E_{1,1}\right)=1 .
$$

Next, we show that $\operatorname{tr}_{M}^{\lambda}$ has indeed the properties of a trace. In order to show that the trace is symmetric, we need an additional lemma first, which can be proven by a small calculation.

Lemma 3.7. Let $A$ be an $\mathbb{K}$-algebra, and let $M$ and $N$ be left $A$-modules. Define a linear map

$$
\begin{aligned}
\xi:\left(M^{*} \otimes_{A} N\right) \times\left(N^{*} \otimes_{A} M\right) & \rightarrow M^{*} \otimes_{A} M \\
(f \otimes n, g \otimes m) & \mapsto f \otimes g(n) . m
\end{aligned}
$$


Then, the following diagram commutes:

$$
\begin{aligned}
&\left(M^{*} \otimes_{A} N\right) \times\left(N^{*} \otimes_{A} M\right) \stackrel{\xi}{\longrightarrow} M^{*} \otimes_{A} M \\
& \qquad \Psi_{M, N} \times \Psi_{N, M} \downarrow \Psi_{M, M} \\
& \operatorname{Hom}_{A}(M, N) \times \operatorname{Hom}_{A}(N, M) \stackrel{\circ}{\longrightarrow} \operatorname{Hom}_{A}(M, M) .
\end{aligned}
$$

Here, the horizontal map at the bottom is given by composition of morphisms of A-modules and $\Psi_{M, M}$ is defined as in equation (3.3).

We are now ready to show that the trace is symmetric:

Lemma 3.8. Let $(A, \lambda)$ be a semisimple, symmetric Frobenius algebra. Let $M$ and $N$ be finitely-generated $A$-modules, and let $f: M \rightarrow N$ and $g: N \rightarrow M$ be morphisms of A-modules. Then,

$$
\operatorname{tr}_{M}^{\lambda}(g \circ f)=\operatorname{tr}_{N}^{\lambda}(f \circ g) .
$$

Proof. Suppose that

$$
\begin{aligned}
& \Psi_{M, N}^{-1}(f)=\sum_{i, j} m_{i}^{*} \otimes n_{j} \in M^{*} \otimes_{A} N \quad \text { and } \\
& \Psi_{N, M}^{-1}(g)=\sum_{k, l} x_{k}^{*} \otimes y_{l} \in N^{*} \otimes_{A} M .
\end{aligned}
$$

We calculate:

$$
\begin{aligned}
\operatorname{tr}_{M}^{\lambda}(g \circ f) & =\left(\lambda \circ \mathrm{ev} \circ \Psi_{M, M}^{-1}\right)(g \circ f) \\
& =(\lambda \circ \mathrm{ev})\left(\sum_{i, j, k, l} m_{i}^{*} \otimes x_{k}^{*}\left(n_{j}\right) \cdot y_{l}\right) \quad \text { (by lemma 3.7) } \\
& =\lambda\left(\sum_{i, j, k, l} m_{i}^{*}\left(x_{k}^{*}\left(n_{j}\right) \cdot y_{l}\right)\right) \\
& =\sum_{i, j, k, l} \lambda\left(x_{k}^{*}\left(n_{j}\right) \cdot m_{i}^{*}\left(y_{l}\right)\right) .
\end{aligned}
$$

On the other hand,

$$
\begin{aligned}
\operatorname{tr}_{N}^{\lambda}(f \circ g) & =\left(\lambda \circ \mathrm{ev} \circ \Psi_{N, N}^{-1}\right)(f \circ g) \\
& =(\lambda \circ \mathrm{ev})\left(\sum_{i, j, k, l} x_{k}^{*} \otimes m_{i}^{*}\left(y_{l}\right) \cdot n_{j}\right) \quad \text { (by lemma 3.7) } \\
& =\lambda\left(\sum_{i, j, k, l} x_{k}^{*}\left(m_{i}^{*}\left(y_{l}\right) \cdot n_{j}\right)\right) \\
& =\sum_{i, j, k, l} \lambda\left(m_{i}^{*}\left(y_{l}\right) \cdot x_{k}^{*}\left(n_{j}\right)\right) .
\end{aligned}
$$

Since $\lambda$ is symmetric, the right hand-sides of equations (3.16) and (3.17) agree. This shows that the trace is symmetric.

Next, we would like to show that the trace is non-degenerate. We first recall a preparatory lemma.

Lemma 3.9 (Koc03, Lemma 2.2.11]). Let $(A, \lambda)$ be a symmetric Frobenius algebra. Then, every other symmetric Frobenius form on $A$ is given by multiplication with a central invertible element of $A$.

We are now ready to show that the trace is non-degenerate.

Lemma 3.10. Let $(A, \lambda)$ be a semisimple, symmetric Frobenius algebra, and let $M$ and $N$ be finitelygenerated A-modules. Then, the bilinear pairing of vector spaces induced by the trace in definition 3.3

$$
\langle-,-\rangle: \operatorname{Hom}_{A}(M, N) \times \operatorname{Hom}_{A}(N, M) \rightarrow \mathbb{K}
$$

is non-degenerate.

$$
(f, g) \mapsto \operatorname{tr}_{M}^{\lambda}(g \circ f)
$$


Proof. By Artin-Wedderburn's theorem, the algebra $A$ is isomorphic to a direct product of matrix algebras over $\mathbb{K}$ :

$$
A \cong \prod_{i=1}^{r} M_{n_{i}}(\mathbb{K}) .
$$

Since the sum of the usual trace of matrices gives each $A$ the structure of a symmetric Frobenius algebra, lemma 3.9 shows that the Frobenius form $\lambda$ of $A$ is given by

$$
\lambda=\sum_{i=1}^{r} \lambda_{i} \operatorname{tr}_{i},
$$

where $\operatorname{tr}_{i}: M_{n_{i}}(\mathbb{K}) \rightarrow \mathbb{K}$ is the usual trace of matrices and $\lambda_{i} \in \mathbb{K}^{*}$ are non-zero scalars.

Recall that a module over a finite-dimensional algebra is finite-dimensional (as a vector space) if and only if it is finitely generated as a module, cf. [SY11, Proposition 2.5]. A classical theorem in representation theory (cf. theorem 3.3.1 in $\left[\mathrm{EGH}^{+} 11\right]$ ) asserts that the only finite-dimensional simple modules of $A$ are given by $V_{1}:=\mathbb{K}^{n_{1}}, \ldots V_{r}:=\mathbb{K}^{n_{r}}$. Since the category $(A \text {-Mod })^{\text {fg }}$ is semisimple, we may decompose the finitely-generated $A$-modules $M$ and $N$ as the direct sum of simple modules:

$$
\begin{aligned}
& M \cong\left(\bigoplus_{i_{1}=1}^{l_{1}} \mathbb{K}^{n_{1}}\right) \oplus\left(\bigoplus_{i_{2}=1}^{l_{2}} \mathbb{K}^{n_{2}}\right) \oplus \cdots \oplus\left(\bigoplus_{i_{r}=1}^{l_{r}} \mathbb{K}^{n_{r}}\right) \\
& N \cong\left(\bigoplus_{i_{1}=1}^{l_{1}^{\prime}} \mathbb{K}^{n_{1}}\right) \oplus\left(\bigoplus_{i_{2}=1}^{l_{2}^{\prime}} \mathbb{K}^{n_{2}}\right) \oplus \cdots \oplus\left(\bigoplus_{i_{r}=1}^{l_{r}^{\prime}} \mathbb{K}^{n_{r}}\right) .
\end{aligned}
$$

By Schur's lemma, any $f \in \operatorname{Hom}_{A}(M, N)$ is given by $f=f_{1} \oplus f_{2} \oplus \ldots \oplus f_{r}$ where $f_{i}$ is a $l_{i}^{\prime} \times l_{i}$-matrix. Similarly, any $g \in \operatorname{Hom}_{A}(N, M)$ is given by $g=g_{1} \oplus g_{2} \oplus \ldots \oplus g_{r}$ where each $g_{i}$ is a $l_{i} \times l_{i}^{\prime}$ matrix. Thus,

$$
\begin{array}{rlr}
\operatorname{tr}_{M}^{\lambda}(g \circ f) & =\operatorname{tr}_{M}^{\lambda}\left(\left(g_{1} f_{1}\right) \oplus\left(g_{2} f_{2}\right) \oplus \ldots \oplus g_{r} f_{r}\right) & \\
& =\sum_{i=1}^{r} \operatorname{tr}_{\left(\mathbb{K}^{\left.n_{i}\right)^{l_{i}}}\right.}^{\lambda}\left(g_{i} f_{i}\right) & \text { (by additivity) } \\
& =\sum_{i=1}^{r} \sum_{j=1}^{l_{i}} \operatorname{tr}_{\mathbb{K}^{n_{i}}}^{\lambda}\left(\left(g_{i} f_{i}\right)_{j, j}\right) & \text { (by lemma 2.12) } \\
& =\sum_{i=1}^{r} \sum_{j=1}^{l_{i}} \sum_{k=1}^{l_{i}^{\prime}} \operatorname{tr}_{\mathbb{K}^{n_{i}}}^{\lambda}\left(\left(g_{i}\right)_{j, k} \circ\left(f_{i}\right)_{k, j}\right) &
\end{array}
$$

Since $f$ was assumed to be non-zero, at least one $\left(f_{i}\right)_{j, k}$ is non-zero. Suppose that $\left(f_{\tilde{i}}\right)_{\tilde{j}, \tilde{k}} \in \operatorname{End}_{A}\left(\mathbb{K}^{n_{i}}\right)$ is not the zero morphism. By Schur's lemma, $\left(f_{\tilde{i}}\right)_{\tilde{j}, \tilde{k}}$ is an isomorphism. Now define $g \in \operatorname{Hom}_{A}(N, M)$ as

$$
\left(g_{i}\right)_{j, k}:=\left(\lambda_{\tilde{i}}\right)^{-1} \delta_{i, \tilde{i}} \delta_{j, \tilde{j}} \delta_{k, \tilde{k}}\left(f_{\tilde{i}}\right)_{\tilde{k}, \tilde{j}}^{-1} .
$$

Then, by example 3.6 .

$$
\operatorname{tr}_{M}^{\lambda}(g \circ f)=\left(\lambda_{\tilde{i}}\right)^{-1} \operatorname{tr}_{\mathbb{K}^{n \tilde{i}}}^{\lambda}\left(\operatorname{id}_{\mathbb{K}^{n \tilde{i}}}\right)=1_{\mathbb{K}} \neq 0
$$

We summarize the situation with the following proposition:

Proposition 3.11. Let $(A, \lambda)$ be a semisimple symmetric Frobenius algebra. Then, the category of finitely-generated A-modules (A-Mod) $)^{\mathrm{fg}}$ has got the structure of a Calabi-Yau category with trace $\operatorname{tr}_{M}^{\lambda}$ : $\operatorname{End}_{A}(M) \rightarrow \mathbb{K}$ as defined in equation (3.5).

Proof. It is well-known that the category of finite-dimensional modules over a finite-dimensional algebra is a finite, linear category, cf. [DSPS14.

If $M$ is a finitely-generated $A$-module, the $\operatorname{trace} \operatorname{tr}^{\lambda}(M): \operatorname{End}(M) \rightarrow \mathbb{K}$ as defined in equation 3.5 is symmetric by lemma 3.8 , while the induced bilinear form is non-degenerate by lemma 3.10. This shows that $(A \text {-Mod })^{\text {fg }}$ is a Calabi-Yau category.

The following example shows that assumption that $A$ is semisimple is a necessary condition. 
Example 3.12 (Counter-example). Let $\mathbb{K}$ be a field of characteristic two, and consider the group algebra $A:=\mathbb{K}\left[\mathbb{Z}_{2}\right]$. Then, $A \cong \mathbb{K}[x] /\left(x^{2}-1\right) \cong \mathbb{K}[x] /(x-1)^{2}$. This is in fact a Frobenius algebra with Frobenius form $\lambda(g)=\delta_{g, e}$, which is not separable. Let $S$ be the trivial representation, and consider a projective two-dimensional representation of $A$ which we shall call $P$. Here, the non-trivial generator $g$ of $A$ acts on $P$ by the matrix

$$
g=\left(\begin{array}{ll}
1 & 1 \\
0 & 1
\end{array}\right)
$$

One easily computes that

$$
\operatorname{Hom}(P, S) \cong\left\{\left(\begin{array}{ll}
0 & b
\end{array}\right) \mid b \in \mathbb{K}\right\}, \quad \text { and } \quad \operatorname{Hom}(S, P) \cong\left\{\left(\begin{array}{l}
a \\
0
\end{array}\right) \mid a \in \mathbb{K}\right\} .
$$

We claim that there is no trace on the representation category of $A$. Indeed, let $\operatorname{tr}_{S}: \operatorname{End}(S) \rightarrow \mathbb{K}$ be any linear map. Then, the pairing

$$
\begin{aligned}
& \operatorname{Hom}(S, P) \otimes \operatorname{Hom}(P, S) \rightarrow \mathbb{K} \\
&\left(\begin{array}{l}
a \\
0
\end{array}\right) \otimes\left(\begin{array}{ll}
0 & b
\end{array}\right) \mapsto \operatorname{tr}_{S}\left(\left(\begin{array}{ll}
0 & b
\end{array}\right)\left(\begin{array}{l}
a \\
0
\end{array}\right)\right)=0
\end{aligned}
$$

is always degenerate. Therefore, a non-degenerate pairing does not exist.

3.2. Constructing the 2-functor Rep ${ }^{\mathrm{fg}}$ on 1-morphisms. The next step of the construction will be the value of Rep ${ }^{\mathrm{fg}}$ on 1-morphisms of Frob, which are compatible Morita contexts. To these, we will have to assign equivalences of Calabi-Yau categories. Let us recall a classical theorem from Morita theory:

Theorem 3.13 ([Bas68, Theorem 3.4 and 3.5]). Let $A$ and $B$ be R-algebras, and let $\left({ }_{B} M_{A},{ }_{A} N_{B}, \varepsilon, \eta\right)$ be a Morita context between $A$ and $B$. Then,

(1) $M$ and $N$ are both finitely-generated and projective as B-modules.

(2) An A-module $X$ is finitely generated over $A$ if and only if $M \otimes_{A} X$ is finitely generated over $B$.

(3) The functor

$$
M \otimes_{A}-: A-\operatorname{Mod} \rightarrow B-\operatorname{Mod}
$$

is an equivalence of linear categories.

This theorem suggests that we should define Rep ${ }^{\mathrm{fg}}$ on Morita contexts by the functor which tensors with the bimodule $M$. In order for this to be well-defined, this functor should be a Calabi-Yau functor as in definition 2.13 if the Morita context is compatible with the Frobenius forms as in definition 2.6 In order to show this, we need an additional lemma:

Lemma 3.14. Let $A$ and $B$ be two semisimple $\mathbb{K}$-algebras. Let $\mathcal{M}=(M, N, \varepsilon, \eta)$ be a Morita context between $A$ and $B$. Write

$$
\varepsilon^{-1}\left(1_{A}\right)=\sum_{i, j} n_{i} \otimes m_{j} \in N \otimes_{B} M
$$

For $T$ a finitely-generated left A-module, define a linear map

$$
\begin{aligned}
\xi: T^{*} \otimes_{A} T & \rightarrow\left(M \otimes_{A} T\right)^{*} \otimes_{B}\left(M \otimes_{A} T\right) \\
t^{*} \otimes t & \mapsto\left(\left(x \otimes y \mapsto \sum_{i} \eta^{-1}\left(x . t^{*}(y) \otimes n_{i}\right)\right) \otimes \sum_{j} m_{j} \otimes t\right)
\end{aligned}
$$

Then, the following diagram commutes.

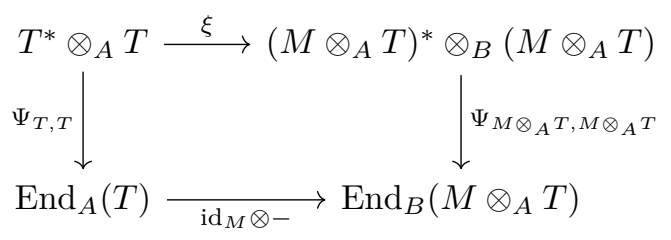

Proof. First note that

$$
\sum_{i, j} \eta^{-1}\left(x \otimes n_{i}\right) \cdot m_{j}=x
$$

for every $x$ in $M$, since $\varepsilon$ and $\eta$ are part of a Morita context. Now, we calculate: 


$$
\left(\operatorname{id}_{M} \otimes-\circ \Psi_{T, T}\right)\left(t^{*} \otimes t\right)(x \otimes y)=\left(\operatorname{id}_{M} \otimes-\right)\left(y \mapsto t^{*}(y) . t\right)(x \otimes y)=x \otimes t^{*}(y) . t
$$

On the other hand,

$$
\begin{aligned}
& \left(\Psi_{M \otimes_{A} T, M \otimes_{A} T} \circ \xi\right)\left(t^{*} \otimes t\right)(x \otimes y)= \\
& =\left(\Psi_{M \otimes_{A} T, M \otimes_{A} T}\right)\left(\left(x \otimes y \mapsto \sum_{i} \eta^{-1}\left(x \cdot t^{*}(y) \otimes n_{i}\right)\right) \otimes \sum_{j} m_{j} \otimes t\right)(x \otimes y) \\
& =\sum_{i, j} \eta^{-1}\left(x \cdot t^{*}(y) \otimes n_{i}\right) \cdot\left(m_{j} \otimes t\right) \\
& =\sum_{i, j} \eta^{-1}\left(x \cdot t^{*}(y) \otimes n_{i}\right) \cdot m_{j} \otimes t \\
& =x \cdot t^{*}(y) \otimes t
\end{aligned}
$$

where in the last line, we have used equation (3.33). This shows that the diagram commutes.

The next proposition shows how the compatibility condition on the Morita context between two Frobenius algebras in definition 2.6 is equivalent to the fact that tensoring with the bimodule $M$ of the Morita context is a Calabi-Yau functor:

Proposition 3.15. Let $\left(A, \lambda^{A}\right)$ and $\left(B, \lambda^{B}\right)$ be two semisimple symmetric Frobenius algebras, and let $(M, N, \varepsilon, \eta)$ be a Morita context between $A$ and $B$. Endow $\operatorname{Rep}^{\mathrm{fg}}(A)$ and $\operatorname{Rep}^{\mathrm{fg}}(B)$ with the Calabi-Yau structure as in definition 3.3 . Then, the Morita context is compatible with the Frobenius forms $\lambda^{A}$ and $\lambda^{B}$ as in definition 2.6 if and only if

$$
\left(M \otimes_{A}-\right): \operatorname{Rep}(A)^{\mathrm{fg}} \rightarrow \operatorname{Rep}(B)^{\mathrm{fg}}
$$

is a Calabi-Yau functor as in definition 2.13 .

Proof. Let ${ }_{A} T$ be a finitely-generated left $A$-module. By definition, the functor $M \otimes_{A}$ - is a Calabi-Yau functor if and only if

$$
\operatorname{tr}_{M \otimes A}^{\lambda^{B}}\left(\mathrm{id}_{M} \otimes f\right)=\operatorname{tr}_{T}^{\lambda^{A}}(f)
$$

for all $f \in \operatorname{End}_{A}(T)$. We have to calculate the left hand-side: Let $f \in \operatorname{End}_{A}(T)$ and write

$$
\Psi_{T, T}^{-1}(f)=\sum_{i, j} t_{i}^{*} \otimes t_{j} \in T^{*} \otimes_{A} T .
$$

Using $n_{i}$ and $m_{j}$ as introduced in formula 3.30 , lemma 3.14 shows that

$$
\begin{aligned}
\Psi_{M \otimes_{A} T, M \otimes_{A} T}^{-1}\left(\operatorname{id}_{M} \otimes f\right) & =\xi \circ \Psi_{T, T}^{-1}(f) \\
& =\sum_{i, j} \xi\left(t_{i}^{*} \otimes t_{j}\right) \\
& =\left(x \otimes y \mapsto \sum_{k, i} \eta^{-1}\left(x \cdot t_{i}^{*}(y) \otimes n_{k}\right)\right) \otimes \sum_{l, j} m_{l} \otimes t_{j} .
\end{aligned}
$$

Hence,

$$
\begin{aligned}
\operatorname{tr}_{M \otimes_{A} T}^{\lambda^{B}}\left(\operatorname{id}_{M} \otimes f\right) & =\left(\lambda^{B} \circ \mathrm{ev} \circ \Psi_{M \otimes_{A} T, M \otimes_{A} T}^{-1}\right)\left(\operatorname{id}_{M} \otimes f\right) \\
& =\sum_{i, j, k, l} \lambda^{B}\left(\eta^{-1}\left(m_{l} \cdot t_{i}^{*}\left(t_{j}\right) \otimes n_{k}\right)\right) .
\end{aligned}
$$

Since

$$
\operatorname{tr}_{T}^{\lambda^{A}}(f)=\sum_{i, j} \lambda^{A}\left(t_{i}^{*}\left(t_{j}\right)\right)
$$

the functor $M \otimes_{A}$ - is a Calabi-Yau functor if and only if the right hand sides of equations (3.40) and (3.41) agree for every $t_{i} \in T^{*}$ and $t_{j} \in T$. Using the fact that the Frobenius forms are symmetric, and thus factor through $A /[A, A]$, this is the case if and only if the Morita context is compatible with the Frobenius forms as in equation 2.9$]$. 
Definition 3.16. Proposition 3.15 enables us to define the 2-functor $\operatorname{Rep}^{\text {fg }}$ on 1 -morphisms of the bicategory Frob: we assign to a compatible Morita context $\mathcal{M}:=(M, N, \varepsilon, \eta)$ between two semisimple symmetric Frobenius algebras $A$ and $B$ the equivalence of Calabi-Yau categories $\operatorname{Rep}^{\mathrm{fg}}(\mathcal{M})$ given by

$$
\operatorname{Rep}^{\mathrm{fg}}(\mathcal{M}):=\left(M \otimes_{A}-\right): \operatorname{Rep}^{\mathrm{fg}}(A) \rightarrow \operatorname{Rep}^{\mathrm{fg}}(B) .
$$

3.3. Constructing the 2-functor $\operatorname{Rep}^{\text {fg }}$ on 2-morphisms. Let $(M, N, \varepsilon, \eta)$ and $\left(M^{\prime}, N^{\prime}, \varepsilon^{\prime}, \eta^{\prime}\right)$ be two compatible Morita contexts between semisimple symmetric Frobenius algebras $A$ and $B$, and let $\alpha: M \rightarrow M^{\prime}$ and $\beta: N \rightarrow N^{\prime}$ be a morphism of Morita contexts. We define a natural transformation $\operatorname{Rep}^{\mathrm{fg}}((\alpha, \beta)):\left(M \otimes_{A}-\right) \rightarrow\left(M^{\prime} \otimes_{A}-\right)$ as follows: for every left $A$-module ${ }_{A} X$, we define the component of the natural transformation as

$$
\operatorname{Rep}^{\mathrm{fg}}((\alpha, \beta))_{X}:=\left(\alpha \otimes \operatorname{id}_{X}\right): M \otimes_{A} X \rightarrow M^{\prime} \otimes_{A} X .
$$

This is indeed a natural transformation because for every morphism $f:{ }_{A} X \rightarrow{ }_{A} Y$ of left $A$-modules, the following diagram

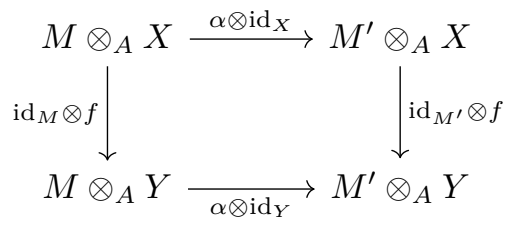

commutes.

Thus, we have obtained the following weak 2 -functor $\operatorname{Rep}^{\text {fg: }}$

$$
\begin{aligned}
\operatorname{Rep}^{\mathrm{fg}}: \text { Frob } & \rightarrow \mathrm{CY} \\
\left(A, \lambda^{A}\right) & \mapsto\left(\operatorname{Rep}^{\mathrm{fg}}(A), \operatorname{tr}^{\lambda^{A}}\right) \\
\left({ }_{B} M_{A},{ }_{B} N_{A}, \varepsilon, \eta\right) & \mapsto\left(M \otimes_{A}-:\left(\operatorname{Rep}^{\mathrm{fg}}(A), \operatorname{tr}^{\lambda^{A}}\right) \rightarrow\left(\operatorname{Rep}^{\mathrm{fg}}(B), \operatorname{tr}^{\lambda^{B}}\right)\right) \\
\left((\alpha, \beta):\left({ }_{B} M_{A},{ }_{B} N_{A}, \varepsilon, \eta\right) \rightarrow\left({ }_{B} M_{A}^{\prime},{ }_{B} N_{A}^{\prime}, \varepsilon^{\prime}, \eta^{\prime}\right)\right) & \mapsto\left(\alpha \otimes \mathrm{id}_{-}:\left(M \otimes_{A}-\right) \rightarrow\left(M^{\prime} \otimes_{A}-\right)\right)
\end{aligned}
$$

Observe that by the definition of the Deligne tensor product, this weak 2-functor is compatible with the symmetric monoidal structures of Frob and CY, and thus is a symmetric monoidal 2-functor.

\section{Proving the EQuivalence}

The aim of this section is to prove that the weak 2 -functor Rep $^{\text {fg }}:$ Frob $\rightarrow$ CY constructed in section 3 is an equivalence of bicategories. This will be done in several steps. First, we show that Rep ${ }^{\mathrm{fg}}$ is essentially surjective. Let $\left(\mathcal{C}, \operatorname{tr}^{\mathcal{C}}\right)$ be a Calabi-Yau category, and let $X_{1}, \ldots, X_{n}$ be representatives of the isomorphism classes of simple objects of $\mathcal{C}$. Define an object $P$ of $\mathcal{C}$ as $P:=\oplus_{i=1}^{n} X_{i}$. Clearly, $A:=\operatorname{End}_{\mathcal{C}}(P)$ is a semisimple, symmetric Frobenius algebra over $\mathbb{K}$ with Frobenius form $\lambda$ given by $\lambda:=\operatorname{tr}_{P}^{\mathcal{C}}$. By proposition 3.11, the category $(A-\operatorname{Mod})^{\mathrm{fg}}$ has the structure of a Calabi-Yau category. We now claim:

Proposition 4.1. The functor

$$
\operatorname{Hom}_{\mathcal{C}}(P,-): \mathcal{C} \rightarrow(A-\operatorname{Mod})^{\mathrm{fg}}
$$

is an equivalence of Calabi-Yau categories.

Proof. An exercise of [EGH $\left.{ }^{+} 11\right]$ which is proven in [DSPS14, Proposition 1.4] asserts that the functor $\operatorname{Hom}_{\mathcal{C}}(P,-)$ is an equivalence of linear categories. Thus, our claim amounts to showing that this functor is compatible with the traces as required in definition 2.13

Write an object $X$ of $\mathcal{C}$ as an arbitrary sum of simple objects, so that $X=\oplus_{j=1}^{m} X_{j}$, and let $f \in$ $\operatorname{End}_{\mathcal{C}}(X)$. Since $\mathcal{C}$ is an additive category, we can represent $f$ as an $m \times m$ matrix

$$
f=\left(\begin{array}{ccc}
f_{1,1} & \ldots & f_{1, m} \\
\vdots & & \vdots \\
f_{m, 1} & \ldots & f_{m, m}
\end{array}\right)
$$

where $f_{k, l} \in \operatorname{Hom}_{\mathcal{C}}\left(X_{l}, X_{k}\right)$. 
Similarly, any $g \in \operatorname{Hom}_{\mathcal{C}}(P, X)$ is naturally a $m \times n$ matrix with entries

$$
g=\left(\begin{array}{ccc}
g_{1,1} & \ldots & g_{1, m} \\
\vdots & & \vdots \\
g_{n, 1} & \ldots & g_{n, m}
\end{array}\right)
$$

where $g_{i, k} \in \operatorname{Hom}_{\mathcal{C}}\left(X_{k}, X_{i}\right)$

Under this identification, $A=\operatorname{End}_{\mathcal{C}}(P)$ acts on $\operatorname{Hom}_{\mathcal{C}}(P, X)$ as $a . f:=f \cdot a$ where $f \cdot a$ is the matrix product of $f$ and $a$.

Then the morphism $\operatorname{Hom}_{\mathcal{C}}(P, f)$ is given by

$$
\begin{aligned}
\operatorname{Hom}_{\mathcal{C}}(P, f): \operatorname{Hom}_{\mathcal{C}}(P, X) & \rightarrow \operatorname{Hom}_{\mathcal{C}}(P, X) \\
g & \mapsto f \cdot g
\end{aligned}
$$

where $f \cdot g$ is the matrix product of $f$ and $g$. As a first step to calculate the trace in $(A-\mathrm{Mod})^{\mathrm{fg}}$, we claim that

$$
\Psi_{\operatorname{Hom}_{\mathcal{C}}(P, X), \operatorname{Hom}_{\mathcal{C}}(P, X)}^{-1}\left(\operatorname{Hom}_{\mathcal{C}}(P, f)\right)=\delta^{*} \otimes \tilde{f},
$$

as an element of $\operatorname{Hom}_{\mathcal{C}}(P, X)^{*} \otimes_{A} \operatorname{Hom}_{\mathcal{C}}(P, X)$, where $\delta^{*} \in \operatorname{Hom}_{\mathcal{C}}(P, X)^{*}$ and $\tilde{f} \in \operatorname{Hom}_{\mathcal{C}}(P, X)$ are defined as follows. First, define the $m \times n$-matrix

$$
\tilde{f}_{k, r}:= \begin{cases}f_{k, r} & \text { if } r \leq m, \\ 0 & \text { else }\end{cases}
$$

Now, given a $m \times n$ matrix $g \in \operatorname{Hom}_{\mathcal{C}}(P, X)$, the element $\delta^{*}(g)$ of $A$ is defined to be an $n \times n$ matrix with entries

$$
\delta^{*}(g)_{r, l}:= \begin{cases}g_{r, l} & \text { if } r \leq m, \\ 0 & \text { else }\end{cases}
$$

Then, if $g \in \operatorname{Hom}_{\mathcal{C}}(P, X)$,

$$
\begin{aligned}
\left(\Psi\left((\delta)^{*} \otimes \tilde{f}\right)(g)\right)_{k, l} & =\left(\delta^{*}(g) \cdot \tilde{f}\right)_{k, l} \\
& =\left(\tilde{f} \cdot \delta^{*}(g)\right)_{k, l} \\
& =\sum_{r=1}^{n} \tilde{f}_{k, r} \cdot \delta^{*}(g)_{r, l} \\
& =\sum_{r=1}^{m} f_{k, r} \circ g_{r, l} \\
& =\left(\operatorname{Hom}_{\mathcal{C}}(P, f)(g)\right)_{k, l}
\end{aligned}
$$

This shows equation 4.5).

We may now calculate the trace of the morphism $\operatorname{Hom}_{\mathcal{C}}(P, f)$ in $(A-\mathrm{Mod})^{\mathrm{fg}}$. By definition of the trace in $(A \text {-Mod })^{\mathrm{fg}}$, we have

$$
\operatorname{tr}_{\operatorname{Hom}_{\mathcal{C}}(P, X)}^{\lambda}\left(\operatorname{Hom}_{\mathcal{C}}(P, f)\right)=\left(\operatorname{tr}_{P} \circ \mathrm{ev} \circ \Psi_{\operatorname{Hom}_{\mathcal{C}}(P, X), \operatorname{Hom}_{\mathcal{C}}(P, X)}^{-1}\right)\left(\operatorname{Hom}_{\mathcal{C}}(P, f)\right) .
$$

Then,

$$
\begin{array}{rlrl}
\operatorname{tr}_{\operatorname{Hom}_{\mathcal{C}}(P, X)}\left(\operatorname{Hom}_{\mathcal{C}}(P, f)\right) & =\left(\operatorname{tr}_{P} \circ \mathrm{ev}\right)\left(\delta^{*} \otimes \tilde{f}\right) & & \text { (by equation } 4.5 \text { ) } \\
& =\operatorname{tr}_{P}\left(\delta^{*}(\tilde{f})\right) & & \text { (by lemma 2.12 } \\
& =\sum_{i=1}^{n} \delta^{*}(\tilde{f})_{i i} & \\
& =\sum_{i=1}^{m} f_{i, i} & & \\
& =\operatorname{tr}_{X}^{\mathcal{C}}(f) . & & \text { (by lemma 2.12 }
\end{array}
$$

This shows that $\operatorname{Hom}_{\mathcal{C}}(P,-)$ is a Calabi-Yau functor.

Next, we follow the exposition in Bas68, Proposition 3.1] and show that the functor Rep ${ }^{\mathrm{fg}}$ is essentially surjective on 1-morphisms. In detail: 
Proposition 4.2. Let $\left(A, \lambda^{A}\right)$ and $\left(B, \lambda^{B}\right)$ be two semisimple, symmetric Frobenius algebras. Endow $(A-\operatorname{Mod}-A)^{\mathrm{fg}}$ and $(B-\mathrm{Mod})^{\mathrm{fg}}$ with the Calabi-Yau structure described in proposition 3.11, and let

$$
F:(A-\mathrm{Mod})^{\mathrm{fg}} \rightleftarrows(B-\mathrm{Mod})^{\mathrm{fg}}: G
$$

be an equivalence of Calabi-Yau categories.

Then, there is a compatible Morita context $\mathcal{M}$ between $A$ and $B$, so that $\operatorname{Rep}^{\mathrm{fg}}(\mathcal{M}) \cong F$.

Proof. The proof works essentially by an application of Eilenberg-Watts and by checking that everything is compatible with the traces: define a $(B, A)$-bimodule $M$ as $M:=F(A)$ which is naturally a left $B$-module, and a right $A$-module by using the map

$$
A \cong \operatorname{End}_{A}(A) \stackrel{F}{\rightarrow} \operatorname{End}_{B}(M) .
$$

The Eilenberg-Watts theorem then shows that the functor $F$ is naturally isomorphic to $M \otimes_{A}-$ (cf. theorem 1 in Wat60). Thus $F$ is a Calabi-Yau functor if and only if $M \otimes_{A}-$ is a Calabi-Yau functor. Similarly, there is an $(A, B)$-bimodule $N$ given by $N:=G(B)$, so that the functor $G$ is naturally isomorphic to $N \otimes_{B}-$.

Furthermore, there are isomorphisms of bimodules

$$
\begin{aligned}
& \varepsilon: N \otimes_{B} M \cong G(M) \cong G\left(M \otimes_{A} A\right) \cong G(F(A)) \cong A \\
& \eta: B \cong F(G(B)) \cong F\left(N \otimes_{B} B\right) \cong F(N) \cong M \otimes_{A} N
\end{aligned}
$$

since $F$ and $G$ is an equivalence of categories. We claim that we can choose these isomorphisms in such a way that $(M, N, \varepsilon, \eta)$ becomes a Morita context.

Indeed, by lemma 2.3 it suffices to show that diagram (2.4) commutes. Let $r_{B}: N \otimes_{B} B \rightarrow N$ be right-multiplication, and let $l_{A}: A \otimes_{B} N \rightarrow N$ be left-multiplication.

Since $\varepsilon, \eta, r_{B}$ and $l_{A}$ are isomorphisms of bimodules, there is a $u \in \operatorname{Aut}_{(A, B)}(N)$, so that

$$
r_{B} \circ \operatorname{id}_{N} \otimes \eta^{-1}=u \circ l_{A} \circ \varepsilon \otimes \operatorname{id}_{N} .
$$

In particular,

$$
u \in \operatorname{Hom}_{A}(N, N) \cong \operatorname{Hom}_{A}(G(B), G(B)) \cong \operatorname{Hom}_{B}(B, B) .
$$

Since every morphism of left $B$-modules $u \in \operatorname{Hom}_{B}(B, B)$ is given by right multiplication with an element of $B$, we may identify $u$ with this element. Since $u$ is also a morphism of right $B$-modules, the element $u$ is in the center of $B$.

Now define an isomorphism of $(B, B)$-bimodules

$$
\begin{aligned}
\tilde{\eta}^{-1}: M \otimes_{A} N & \rightarrow B \\
m \otimes n & \mapsto u . \eta^{-1}(n \otimes m) .
\end{aligned}
$$

Now, if we replace $\eta$ by $\tilde{\eta}$ we have made diagram (2.3) commute. Thus, $(M, N, \varepsilon, \tilde{\eta})$ is a Morita context, which is compatible with the Frobenius forms by proposition 3.15

We are now ready to prove the following theorem:

Theorem 4.3. The weak 2-functor $\operatorname{Rep}^{\mathrm{fg}}: \mathrm{Frob} \rightarrow \mathrm{CY}$ is an equivalence of bigroupoids.

Proof. In a first step, proposition 3.11 shows the representation category of a semisimple symmetric Frobenius algebra has indeed got the structure of a Calabi-Yau category. Furthermore, this assignment is essentially surjective on objects: given a Calabi-Yau category $\mathcal{C}$, proposition 4.1 shows how to construct a Frobenius algebra $A$ so that $\operatorname{Rep}^{\mathrm{fg}}(A)$ and $\mathcal{C}$ are equivalent as Calabi-Yau categories.

Now, given a compatible Morita context between two semisimple symmetric Frobenius algebras $A$ and $B$, proposition 3.15 shows how to construct a Calabi-Yau functor between the representation categories. Furthermore, this assignment is essentially surjective by proposition 4.2

Finally, one shows by hand that Rep ${ }^{\mathrm{fg}}$ induces a bijection on 2-morphisms of Frob and CY which carry no additional structures or properties.

Remark 4.4. Note that both Frob and CY have an additional symmetric monoidal structure, which is preserved by the 2-functor Rep ${ }^{\mathrm{fg}}$. Essentially, this follows from the definition of the Deligne tensor product, and by observing that definition 3.3 of the Calabi-Yau structure on the representation category of a semisimple symmetric Frobenius algebra is well-behaved under taking tensor products. Thus, $\operatorname{Rep}^{\mathrm{fg}}$ : Frob $\rightarrow \mathrm{CY}$ is even an equivalence of symmetric monoidal bigroupoids. 
Remark 4.5. Let us also comment on the relationship between the weak 2-functor $\operatorname{Rep}^{\text {fg }}:$ Frob $\rightarrow \mathrm{CY}$ in theorem 4.3 and the homotopy fixed points of a $G$-action on a bicategory as considered in [HSV16]: by [HSV16, Corollary 4.2 and 4.5] there are equivalences of bigroupoids

$$
\begin{aligned}
& \mathscr{K}\left(\mathrm{Alg}_{2}^{\mathrm{fd}}\right)^{S O(2)} \cong \text { Frob } \\
& \mathscr{K}\left(\operatorname{Vect}_{2}^{\mathrm{fd}}\right)^{S O(2)} \cong \mathrm{CY}
\end{aligned}
$$

where $\mathscr{K}\left(\mathrm{Alg}_{2}^{\mathrm{fd}}\right)^{S O(2)}$ and $\mathscr{K}\left(\mathrm{Vect}_{2}^{\mathrm{fd}}\right)^{S O(2)}$ are the bigroupoids of homotopy fixed points of the trivial $S O(2)$-action on the core of fully-dualizable objects of $\mathrm{Alg}_{2}$ and $\mathrm{Vect}_{2}$. Now, as the $S O(2)$-action is trivial, the weak 2-functor Rep $: \mathscr{K}\left(\mathrm{Alg}_{2}^{\mathrm{fd}}\right) \rightarrow \mathscr{K}\left(\operatorname{Vect}_{2}^{\mathrm{fd}}\right)$ sending a semisimple algebra to its category

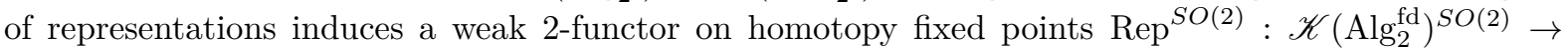
$\mathscr{K}\left(\operatorname{Vect}_{2}^{\mathrm{fd}}\right)^{S O(2)}$. One now checks that this induced 2-functor on homotopy fixed points is pseudonaturally isomorphic to the weak 2 -functor $\operatorname{Rep}^{\text {fg }}$ of theorem 4.3 . More precisely, the following diagram commutes up to pseudo-natural isomorphism:

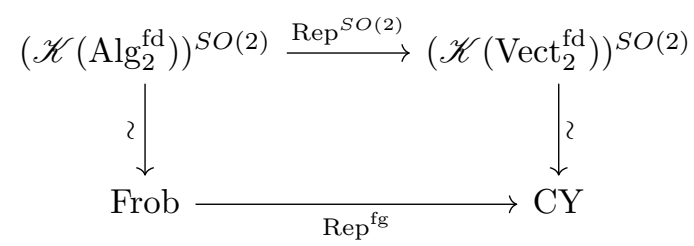

The commutativity of the diagram boils down to a Schur's lemma type of argument with a vector of invertible scalars after following the construction in HSV16]. Details of the construction are given in the appendix and in Hes17.

\section{APPENDIX A. INDUCED FUNCTORS ON HOMOTOPY FIXED POINTS}

So far, we have constructed an equivalence of bicategories $\operatorname{Rep}^{\text {fg }}:$ Frob $\rightarrow$ CY. In this section, we show that this equivalence is actually the "equivariantization" of the 2-functor sending an algebra to its category of modules.

In order to do so, we introduce the notion of a the "equivariantization" of a weak 2-functor between bicategories equipped with a $G$-action, where $G$ is a topological group. Let us briefly recall the relevant definitions: For a group $G$, we denote with $B G$ the category with one object and $G$ as morphisms. Similarly, if $\mathcal{C}$ is a monoidal (bi-)category, $B \mathcal{C}$ will denote the (tri-)bicategory with one object and $\mathcal{C}$ as endomorphism (bi-)category of this object.

For a topological group $G$, let $\Pi_{2}(G)$ be its fundamental 2-groupoid, and $B \Pi_{2}(G)$ the tricategory with one object called $*$ and $\Pi_{2}(G)$ as endomorphism bicategory. A $G$-action on a bicategory $\mathcal{C}$ is then defined to be a trifunctor $\rho: B \Pi_{2}(G) \rightarrow$ Bicat with $\rho(*)=\mathcal{C}$, where Bicat is the tricategory of bicategories. Furthermore, given a $G$-action $\rho$ on a bicategory, we define the bicategory of homotopy fixed points $\mathcal{C}^{G}$ to be the bicategory $\operatorname{Nat}(\Delta, \rho)$ where objects are given by tritransformations between the constant functor $\Delta$ and $\rho$, 1-morphisms are modifications, and 2-morphisms are perturbations. In the following, we will use the notation of [HSV16] concerning homotopy fixed points.

Definition A.1. Let $\rho: B \Pi_{2}(G) \rightarrow$ Bicat be a $G$-action on the bicategory $\rho(*)=\mathcal{C}$, and let $\rho^{\prime}$ : $B \Pi_{2}(G) \rightarrow$ Bicat be a $G$-action on $\rho^{\prime}(*)=\mathcal{D}$. Let $H: \mathcal{C} \rightarrow \mathcal{D}$ be a weak 2 -functor. A $G$-equivariant structure for the weak 2-functor $H: \mathcal{C} \rightarrow \mathcal{D}$ consists of:

- a pseudo-natural transformation $T$ in the diagram

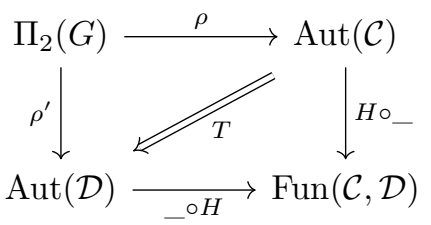

which very explicitly consists of the data:

- a pseudo-natural transformation

$$
T_{g}: H \circ F_{g} \rightarrow F_{g}^{\prime} \circ H
$$

for every $g \in G$, explaining the name $G$-equivariant structure, 
- For every path $\gamma: g \rightarrow h$, an invertible modification $T_{\gamma}$ in the diagram

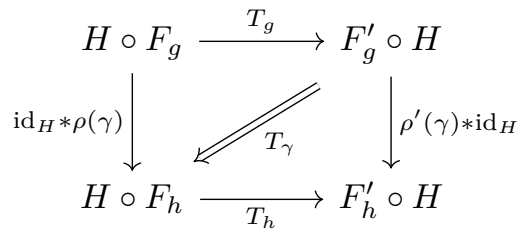

- for every $g, h \in G$, invertible modifications $P_{g h}$

(A.4)

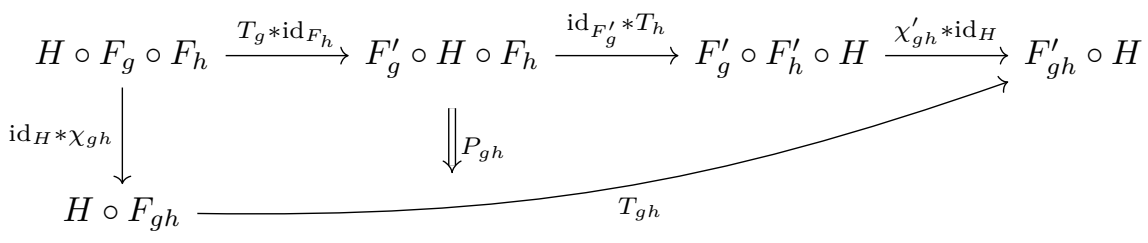

- a modification $N$

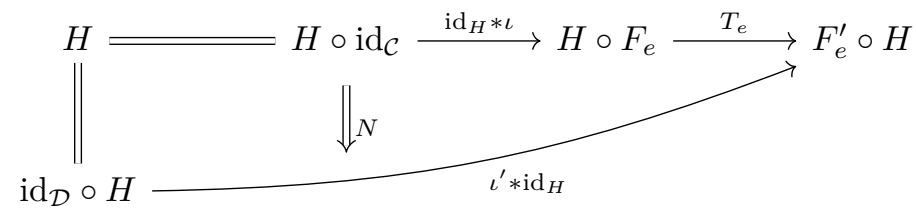

so that the three equations of a tritransformation in definition 3.3 of [GPS95] are fulfilled.

Remark A.2. We have defined a $G$-equivariant structure on a weak 2 -functor $H$ in such a way that it induces a tritransformation $\rho \rightarrow \rho^{\prime}$ between the two actions. It is crucial to remark that the $G$-equivariant structure induces a weak 2-functor $H^{G}$ on homotopy fixed point bicategories:

$$
H^{G}: \mathcal{C}^{G}=\operatorname{Nat}(\Delta, \rho) \rightarrow \operatorname{Nat}\left(\Delta, \rho^{\prime}\right)=\mathcal{D}^{G} .
$$

Explicitly, the induced functor on homotopy fixed points is given as follows:

Definition A.3. Suppose that $H: \mathcal{C} \rightarrow \mathcal{D}$ is a weak 2 -functor between bicategories endowed with $G$-actions $\rho$ and $\rho^{\prime}$, and suppose that $H$ possesses a $G$-equivariant structure as in definition A.1 Then, the induced functor $H^{G}: \mathcal{C}^{G} \rightarrow \mathcal{D}^{G}$ is then given as follows: On objects $(c, \Theta, \Pi, M)$ as defined in HSV16, Remark 3.11] of the homotopy fixed point bicategory $\mathcal{C}^{G}$ we define:

- On the object $c$ of $\mathcal{C}$, we have $H^{G}(c):=H(c)$,

- On the pseudo-natural equivalence $\Theta$, we define the functor on the 1-cell $\Theta_{g}: c \rightarrow F_{g}(c)$ by

$$
H^{G}\left(\Theta_{g}\right):=\left(H(c) \stackrel{H\left(\Theta_{g}\right)}{\longrightarrow} H\left(F_{g}(c)\right) \stackrel{T_{g}(c)}{\longrightarrow} F_{g}^{\prime}(H(c))\right),
$$

where $F_{g}$ and $F_{g}^{\prime}$ are data given by the action as defined in [HSV16, Remark 3.8], whereas on the 2-dimensional component $\Theta_{\gamma}$ in the diagram

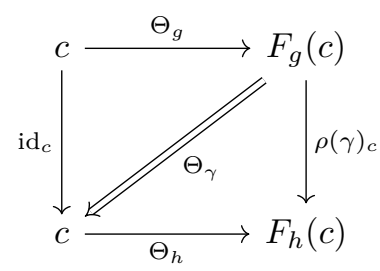

we assign the 2-morphism

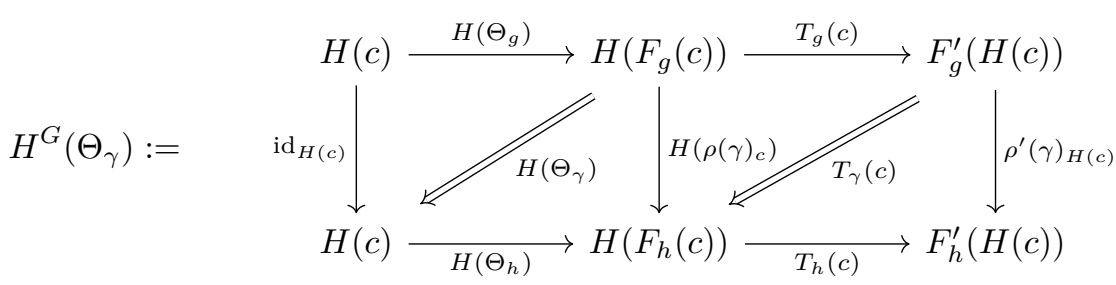

- For the modification $\Pi$, we assign the 2-morphism 
(A.10)

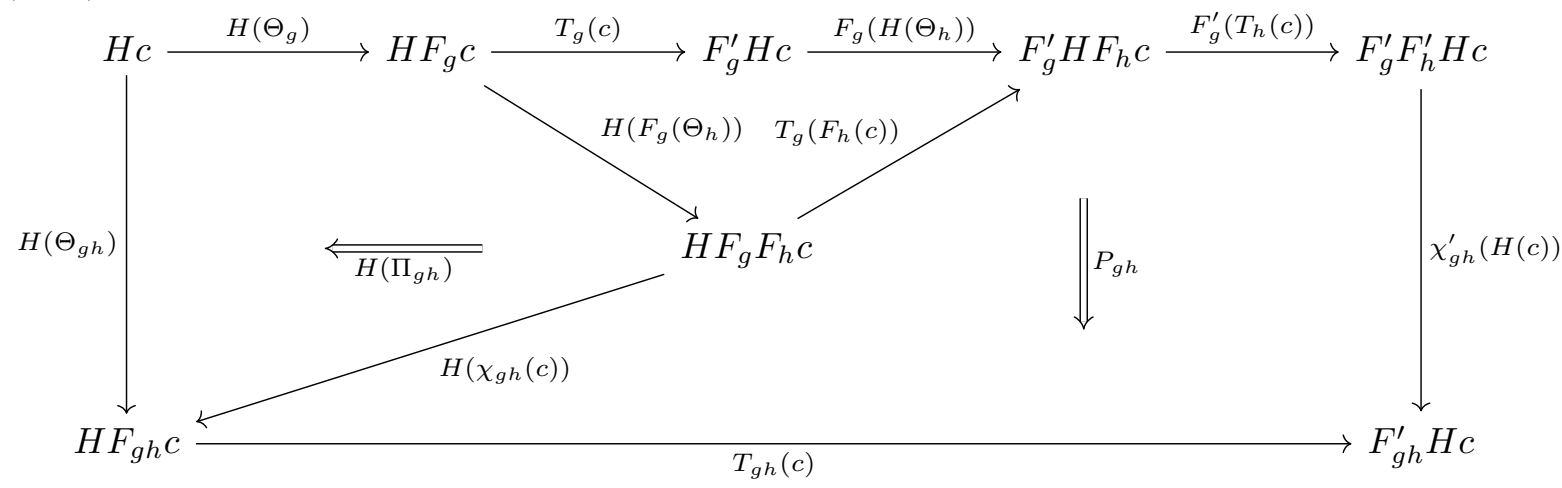

- The induced functor sends the modification $M$ to the modification in the diagram

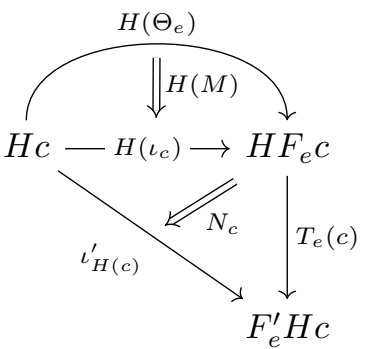

It is a straightforward, but tedious to see that $\left(H^{G}(c), H^{G}(\Theta), H^{G}(\Pi), H^{G}(M)\right)$ is a homotopy fixed point in $\mathcal{D}^{G}$.

Definition A.4. If $(f, m):(c, \Theta, \Pi, M) \rightarrow(\tilde{c}, \tilde{\Theta}, \tilde{\Pi}, \tilde{M})$ is a morphism of homotopy fixed points in $\mathcal{C}^{G}$ as considered in HSV16. Remark 3.12], the induced functor $H^{G}$ is given on 1-morphisms of homotopy fixed points by $H^{G}(f):=H(f)$, and by

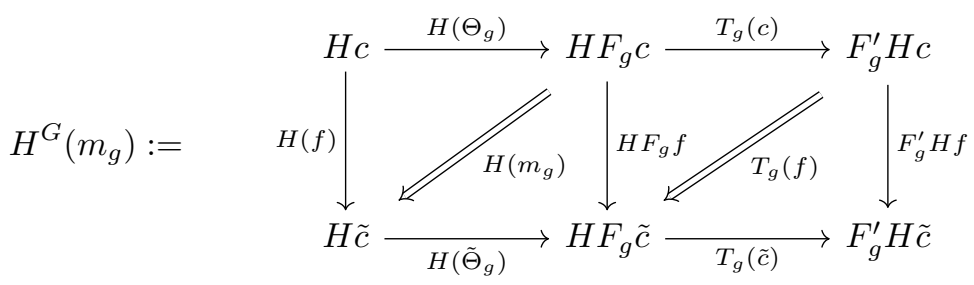

Definition A.5. If $(f, m)$ and $(\xi, n)$ are two 1-morphisms of homotopy fixed points in $\mathcal{C}^{G}$, and $\sigma$ : $((f, m) \rightarrow(\xi, n))$ is a 2-morphism of homotopy fixed points as in [HSV16, Remark 3.14], the induced functor on 2-morphisms is given by $H^{G}(\sigma):=H(\sigma)$.

Lemma A.6. Let Rep : $\mathscr{K}\left(\operatorname{Alg}_{2}^{\mathrm{fd}}\right) \rightarrow \mathscr{K}\left(\mathrm{Vect}_{2}^{\mathrm{fd}}\right)$ be the functor which send a finite-dimensional, semisimple algebra to its category of finitely-generated modules, and let $\rho: \Pi_{2}(S O(2)) \rightarrow \operatorname{Aut}\left(\mathscr{K}\left(\operatorname{Alg}_{2}^{\mathrm{fd}}\right)\right)$ and $\rho^{\prime}: \Pi_{2}(S O(2)) \rightarrow \operatorname{Aut}\left(\mathscr{K}\left(\operatorname{Vect}_{2}^{\mathrm{fd}}\right)\right)$ be the trivial actions. Then, Rep has got a canonical $S O(2)$ equivariant structure given by taking identities everywhere.

Proof. We need to provide the data in definition A.1. Since both actions are trivial, we may choose $T_{g}:$ Rep $\rightarrow$ Rep to be the identity pseudo-natural transformation for every $g \in G$, and $T_{\gamma}: \operatorname{id}_{\operatorname{Rep}} \circ T_{g} \rightarrow$ $T_{h} \circ$ id $_{\text {Rep }}$ to be the identity modification for every path $\gamma: g \rightarrow h$. Furthermore, we may also choose $P_{g h}$ and $N$ to be the identity modifications.

Since the representation functor is $S O(2)$-equivariant, it induces a functor on homotopy fixed point bicategories by definitions A.3 A.4 and A.5 We claim:

Theorem A.7. The diagram

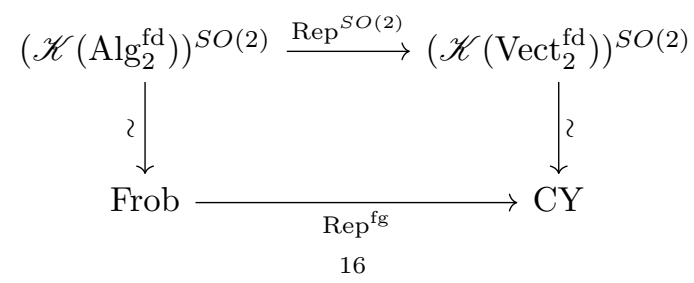


commutes up to a pseudo-natural isomorphism. Here, the unlabeled equivalences are induced by [HSV16, Corollary 4.2 and 4.5], while the functor $\operatorname{Rep}^{\mathrm{fg}}$ is constructed in section 3 .

Proof. Let

$$
\begin{aligned}
F:\left(\mathscr{K}\left(\mathrm{Alg}_{2}^{\mathrm{fd}}\right)\right)^{S O(2)} & \cong \text { Frob } \\
G:\left(\mathscr{K}\left(\mathrm{Vect}_{2}^{\mathrm{fd}}\right)\right)^{S O(2)} & \cong \mathrm{CY}
\end{aligned}
$$

be the equivalences of bicategories constructed in [HSV16. By [HSV16, Theorem 4.1], the bicategory $\left(\mathscr{K}\left(\operatorname{Alg}_{2}^{\mathrm{fd}}\right)\right)^{S O(2)}$ is equivalent to a bicategory where objects are given by semisimple algebras $A$, together with an isomorphism of Morita contexts $\lambda: \operatorname{id}_{A} \rightarrow \operatorname{id}_{A}$, where $\operatorname{id}_{A}$ is the identity Morita context, consisting of the algebra $A$ considered as an $(A, A)$-bimodule. If $(A, \lambda)$ is an object of $\left(\mathscr{K}\left(\operatorname{Alg}_{2}^{\mathrm{fd}}\right)\right)^{S O(2)}$, we need to construct an equivalence of Calabi-Yau categories

$$
\eta_{(A, \lambda)}:\left(G \circ \operatorname{Rep}^{S O(2)}\right)(A, \lambda) \rightarrow\left(\operatorname{Rep}^{\mathrm{fg}} \circ F\right)(A, \lambda) .
$$

By definition A.3 the value of $\operatorname{Rep} S O(2)$ on $A$ is given by $\operatorname{Rep}^{\mathrm{fg}}(A)$, the category of finitely-generated modules of $A$. The value of $\operatorname{Rep}^{S O(2)}$ on $\lambda$ is given by the natural isomorphism defined as follows: if ${ }_{A} M$ is an $A$-module, the natural transformation $\operatorname{Rep}^{S O(2)}(\lambda)$ of the identity functor on $\operatorname{Rep}^{\mathrm{fg}}(A)$ is given in components by

$$
\operatorname{Rep}^{S O(2)}(\lambda)_{M}:=\left(M \cong A \otimes_{A} M \stackrel{\lambda \otimes \mathrm{id}_{M}}{\longrightarrow} A \otimes_{A} M \cong M\right) .
$$

We know that $A$ is isomorphic to a direct sum of matrix algebras:

$$
A \cong \bigoplus_{i=1}^{r} M_{d_{i}}(\mathbb{K})
$$

Let

$$
\left(\lambda_{1}, \ldots, \lambda_{r}\right) \in \mathbb{K}^{r} \cong Z(A) \cong \operatorname{End}_{(A, A)}(A)
$$

be the scalars corresponding to the isomorphism of Morita contexts $\lambda$. Then, the Calabi-Yau structure on $\left(G \circ \operatorname{Rep}^{S O(2)}\right)(A, \lambda)$ is given as follows: it suffices to write down a trace for the simple modules, because $\operatorname{Rep}^{\mathrm{fg}}(A)$ is semisimple. If $X_{i}$ is a simple $A$-module, chasing through the equivalence $G$ shows that the trace is given by identifying the division algebra $\operatorname{End}_{\operatorname{Rep}^{\mathrm{fg}}(A)}\left(X_{i}\right)$ with the algebraically closed ground field $\mathbb{K}$ by Schur's lemma, and then (up to a permutation of the simple modules) multiplying with the scalar $\lambda_{i}$.

On the other hand, chasing through the equivalence of bicategories $F$ in [HSV16. Corollary 4.2], we see that the Frobenius algebra $F(A, \lambda)$ is given by the semisimple algebra $A$ as in equation (A.17), together with the Frobenius form given by taking direct sums of matrix traces, multiplied with the scalars $\lambda_{i}$ in equation A.18). Using the construction of the functor $\operatorname{Rep}^{\mathrm{fg}}$ in section 3 shows that the CalabiYau category $\left(\operatorname{Rep}^{\mathrm{fg}} \circ F\right)(A, \lambda)$ is given by the linear category $\operatorname{Rep}^{\mathrm{fg}}(A)$, together with the Calabi-Yau structure given by the composite of the Frobenius form with the Hattori-Stallings trace. For a simple module $X_{i}$, this Calabi-Yau structure is given by multiplying with the scalar $\lambda_{i}$ under the identification $\operatorname{End}_{\operatorname{Rep}^{\mathrm{fg}}(A)}\left(X_{i}\right) \cong \mathbb{K}$. Thus, we have succeeded in finding an equivalence $\eta$ as required in equation A.15. Going through the equivalences $F$ and $G$, we check that $\eta$ is even pseudo-natural. This shows that the diagram A.13 commutes up to a pseudo-natural isomorphism. 


\section{REFERENCES}

[AW92] W. A. Adkins and S. Weintraub, Algebra: An approach via module theory, Graduate Text in Mathematics, Springer New York, 1992.

[Bas68] H. Bass, Algebraic K-theory, W. A. Benjamin, Inc., New York-Amsterdam, 1968.

[Bas76] - Euler characteristics and characters of discrete groups, Invent. Math. 35 (1976), no. 1, 155-196.

[BDSV15] B. Bartlett, C. L. Douglas, C. J. Schommer-Pries, and J. Vicary, Modular categories as representations of the 3-dimensional bordism 2-category. arXiv:1509.06811

[Dav11] O. Davidovich, State sums in two dimensional fully extended topological field theories, Ph.D. thesis, University Texas at Austin (2011).

[DSPS14] C. L. Douglas, C. Schommer-Pries, and N. Snyder, The balanced tensor product of module categories, 2014. arXiv:1406.4204

$\left[\mathrm{EGH}^{+} 11\right]$ P. Etingof, O. Goldberg, S. Hensel, T. Liu, A. Schwender, D. Vaintrob, and E. Yudovina, Introduction to representation theory, Student mathematical library, vol. 59, American Mathematical Society, 2011.

[FHLT10] D. S. Freed, M. J. Hopkins, J. Lurie, and C. Teleman, Topological quantum field theories from compact Lie groups, A celebration of the mathematical legacy of Raoul Bott, CRM Proc. Lecture Notes, vol. 50, Amer. Math. Soc., Providence, RI, 2010, pp. 367-403. arXiv:0905.0731

[Hat65] A. Hattori, Rank element of a projective module, Nagoya Math. J. 25 (1965), 113-120.

[Hes17] J. Hesse, Group actions on bicategories and topological quantum field theories, Ph.D. thesis, Universität Hamburg, 2017.

[HSV16] J. Hesse, C. Schweigert, and A. Valentino, Frobenius algebras and homotopy fixed points of group actions on bicategories, ArXiv e-prints (2016). arXiv:1607.05148

[Koc03] J. Kock, Frobenius algebras and 2D topological quantum field theories, London Mathematical Society, Student Texts 59, Cambridge University Press, 2003.

[GPS95] R. Gordon, A. J. Power, and R. Street, Coherence for tricategories, Mem. Amer. Math. Soc. 117 (1995), no. 558.

[Lam12] T. Y. Lam, Lectures on modules and rings, Graduate Texts in Mathematics, Springer New York, 2012.

[Lur09] J. Lurie, On the classification of topological field theories, Current developments in mathematics, Int. Press, Somerville, MA, 2009, pp. 129-280.

[MS06] G. W. Moore and G. Segal, D-branes and K-theory in 2D topological field theory, 2006. arXiv:hep-th/0609042

[Sch13] G. Schaumann, Traces on module categories over fusion categories, J. Algebra 379 (2013), $382-425$. arXiv:1206.5716

[SP09] C. J. Schommer-Pries, The classification of two-dimensional extended topological field theories, ProQuest LLC, Ann Arbor, MI, 2009, Thesis (Ph.D.)-University of California, Berkeley. arXiv:1112.1000

[Sta65] J. Stallings, Centerless groups - an algebraic formulation of Gottlieb's theorem, Topology 4 (1965), no. 2, 129 134 .

[SY11] A. Skowroński and K. Yamagata, Frobenius algebras I, EMS Textbooks in Mathematics, European Mathematical Society, Zürich, 2011, Basic representation theory.

[Wat60] C. E. Watts, Intrinsic characterizations of some additive functors, Proc. Amer. Math. Soc. 11 (1960), 5-8.

Fachbereich Mathematik, Universität Hamburg, Bereich Algebra und Zahlentheorie, Bundesstrasse 55, D 20146 HAMBURG 